\title{
Co-culture of human fibroblasts and Borrelia burgdorferi enhances collagen and growth factor mRNA
}

\author{
Elisabeth Aberer ${ }^{1} \cdot$ Milana Surtov-Pudar $^{1} \cdot$ Daniel Wilfinger $^{1} \cdot$ Alexander Deutsch $^{2} \cdot$ Gerd Leitinger $^{3}$. \\ Helmut Schaider ${ }^{1,4}$
}

Received: 9 August 2017 / Revised: 2 November 2017 / Accepted: 17 November 2017 / Published online: 6 December 2017

(c) The Author(s) 2018. This article is an open access publication

\begin{abstract}
Skin fibrosis has been reported in Borrelia burgdorferi infection in Europe, but has been questioned by several authors. The objective of the present study was to examine the interaction of skin fibroblasts with B. burgdorferi sensu stricto B31 (BB) and $B$. afzelii (BA) in vitro by electron microscopy. We also determined the expression of collagen type I, TGF- $\beta$, FGF-1, calreticulin (CALR), decorin (DCN), and PDGF- $\alpha$ at the mRNA level in Borrelia/fibroblast co-cultures. Intact Borrelia attach to and transmigrate fibroblasts, and undergo cystic transformation outside the fibroblasts. Fibroblasts preserve their vitality and express a prominent granular endoplasmic reticulum, suggesting activated protein synthesis. On two different semi-quantitative real-time PCR assays, BB- and BA/fibroblast co-cultures showed a significant induction of type I collagen mRNA after 2 days compared to fibroblasts (fourfold for BA and 1.8-fold for BB; $p<0.02$ ). In addition, there was a significant upregulation of mRNA expression of TGF- $\beta$, CALR, PDGF- $\alpha$, and DCN in BA and BB co-cultures compared to control fibroblasts in monolayer cultures after 2 days $(p<0.01)$. The BA/fibroblast co-culture induced a considerably greater upregulation of collagen and growth factor mRNA compared to BB/fibroblast co-culture. In contrast, a significant down-regulation of FGF-1 (20-fold for BA and 4.5-fold for BB) mRNA expression was detected in co-cultures compared to controls $(p<0.01)$. The results of the study support the hypothesis that BB sensu lato, and BA in particular, enhances collagen mRNA expression and can stimulate growth factors responsible for increased collagen production.
\end{abstract}

Keywords B. burgdorferi - B. afzelii · Fibroblast · Co-culture · mRNA type I collagen · TGF- $\beta$ FGF-1 · PDGF · Decorin · Calreticulin · Fibrosis

\section{Introduction}

Borrelia burgdorferi sensu lato, a bacterium transmitted by ticks into the skin of humans and animals, has a high affinity for connective tissue and binds to decorin, fibronectin,

Elisabeth Aberer

elisabeth.aberer@medunigraz.at

1 Department of Dermatology, Medical University of Graz, Auenbrugger Platz 8, 8036 Graz, Austria

2 Division of Haematology, Internal Medicine, Medical University of Graz, Graz, Austria

3 Research Unit Electron-Microscopic Techniques, Institute of Cell Biology, Histology and Embryology, Medical University of Graz, Graz, Austria

4 The University of Queensland Diamantina Institute, Translational Research Institute, University of Queensland, Brisbane, QLD, Australia integrin, plasminogen, glycosaminoglycans, and other components of connective tissue $[9,28,33]$. Borrelia may invade type I collagen fibres [52] and also fibroblasts, where they are protected from the action of antibiotics such as ceftriaxone [23].

Lyme borreliosis in Europe is caused by three main genospecies of B. burgdorferi sensu lato, namely, B. burgdorferi sensu stricto (BB), B. afzelii (BA), and B. garinii (BG) [29]. Only BB exists in the United States. A certain organotropism of the different genospecies has been observed. BA, the most common detected genospecies in Europe, has a high affinity for the skin. Its persistence causes the chronic infection known as acrodermatitis chronica atrophicans [10,35]. Skin infection of long duration is characterized by atrophy on one hand and skin sclerosis on the other, as seen in ACA with pseudoscleroderma [3]. In the brain, reactive gliosis or astrogliosis evidenced by condensation of astrocytic fibres was observed in the rhesus macaque infected with a neurotropic 
B. burgdorferi strain [37]. Degenerative lesions and fibrosis were seen in peripheral nerves [38]. Experimental studies on monkeys have shown that BB infection of heart tissue also induces fibrosis, as shown by a significantly increased density score [7].

Fibrosis of the skin has been repeatedly observed in association with a Borrelia infection in Europe only, namely, circumscribed scleroderma (cSc), lichen sclerosus et atrophicus, exacerbation of systemic scleroderma, trigger finger, or the carpal tunnel syndrome $[5,6,15,40,48]$. Since sclerotic skin lesions have not been reported in the United States, the question arises as to whether the predominant genospecies in Europe, BA or BG, can influence and exaggerate collagen turnover. In cSc, the BA genospecies was isolated from skin biopsies and DNA of BA- and BG-, but not BB-DNA has been detected in cSc patients' skin biopsies from patients in Germany, Austria, and Japan but not in the United States $[6,11]$.

CSc and SSc are caused by excessive deposits of type I collagen [25], manifested by more than a threefold elevation of collagen I mRNA levels in SSc skin compared to controls [17]. This phenomenon is promoted by several cytokines and growth factors. Isolated scleroderma fibroblasts produce elevated mRNA levels of type I collagen and transforming growth factor (TGF- $\beta$ ) in culture [44]. Furthermore, plateletderived growth factor (PDGF- $\alpha$ ) [21], calreticulin (CALR) $[27,54]$, decorin (DCN) [46], and other molecules are thought to be involved in the increased collagen syntheses [45]. Basic fibroblast growth factor (FGF-1) induces collagen production by stimulating skin fibroblasts and connective tissue growth factor (CTGF) [8].

Based on these reports, we studied the behaviour of fibroblasts in co-culture with different Borrelia genospecies. We looked for a difference between collagen syntheses in co-culture compared to fibroblasts only, induced either by BB or by BA. Our aim was to simultaneously show the interaction of fibroblasts with Borrelia by electron microscopy and investigate the synthesis of mRNA collagen type I as well as the synthesis of different growth factors and molecules known to stimulate collagen production compared to fibroblasts only.

\section{Materials and methods}

\section{Culture of Borrelia}

Three strains of B. burgdorferi, BB strain B31 (gifted by Bettina Wilske of the Max von Pettenkofer Institute in Munich, Germany), and two strains of BA, isolated from skin biopsies of patients with erythema migrans at the department of dermatology, Medical University of Graz (ethical approval No. 181 99/00), were used. The two BA isolates were typed by RFLP analysis at the Institute of
Hygiene and Microbiology, Medical University of Graz, by Doris Stünzner [42].

Borrelia were stored at $-70{ }^{\circ} \mathrm{C}$, thawed at room temperature, and cultured in $8-\mathrm{ml}$ Falcon tubes at $34{ }^{\circ} \mathrm{C}$ in BSK-H medium [36]. Cultures were monitored once a week in regard of growth, vitality, movement of Borrelia, and the purity of the medium by dark-field microscopy. Borrelia were counted in a Petroff-Hausser counting chamber. For subcultures, 100-1000 $\mu \mathrm{l}$ of Borrelia suspension was inoculated in fresh BSK-H medium. Borrelia were subcultured to a density of $10^{8}$ cells/microscopic field.

\section{Culture of fibroblasts}

The FF2462 fibroblast cell line was isolated from human foreskin [provided by Dr. Meenhard Herlyn (The Wistar Institute, Philadelphia, PA, USA)] [49]. The cells were stored at $-70{ }^{\circ} \mathrm{C}$, thawed in warm water, and dissolved in $8 \mathrm{ml}$ DMEM (500 ml DMEM supplemented with $50 \mathrm{ml}$ FCS und $10 \mathrm{ml}$ glutamine). Fibroblasts were centrifuged at $1200 \mathrm{rpm}$ for $5 \mathrm{~min}$, and the pellet was dissolved in $4 \mathrm{ml}$ DMEM and applied to culture tubes of $75 \mathrm{~cm}^{2}$. Ten millilitres of DMEM was then added and the cells were cultured at $37{ }^{\circ} \mathrm{C}$ with $5 \% \mathrm{CO}_{2}$. After 4 days, the supernatant was replaced by $10 \mathrm{ml}$ fresh DMEM and cultured for another 3 days. Subcultures were performed once a week to a cell count of $10^{5}$ cells.

\section{Co-culture of fibroblasts with B. burgdorferi for studying cell morphology by electron microscopy}

Four parallel analyses were started for the co-culture experiments using the BB B31 and the BA 1 strain; the latter showed better growth than BA2. Cultures could be harvested after $24,48,72 \mathrm{~h}$, and 7 days. For the experiments, a polypropylene lattice was placed in each well of a 6-well plate, and then, $10^{5}$ fibroblasts in $2 \mathrm{ml}$ DMEM were pipetted in the well. After $24 \mathrm{~h}$, DMEM was withdrawn and replaced by $1 \mathrm{ml}$ RPMI. After centrifugation of Borrelia cultures at $1500 \mathrm{rpm}$, the pellet was resuspended in $1 \mathrm{ml}$ of RPMI containing $10^{8}$ Borrelia $/ \mathrm{ml}$, added to the wells, and incubated for 24, 48 and $96 \mathrm{~h}$, and 7 days, respectively.

For morphological analysis, the fluid of the plate was withdrawn and the cells were removed from the lattices, fixed for $30 \mathrm{~min}$ in $2.5 \%$ glutaraldehyde in $0.1 \mathrm{M}$ cacodylate buffer $\mathrm{pH} 7.4$ for $2 \mathrm{~h}$, rinsed in cacodylate buffer for $2 \mathrm{~h}$ at $4{ }^{\circ} \mathrm{C}$, and centrifuged at $800 \mathrm{rpm}$ for $5 \mathrm{~min}$. The pellet was placed in a $2 \%$ agarose solution, cooled to $4{ }^{\circ} \mathrm{C}$, and then cut into small blocks which were again fixed in $2.5 \%$ glutaraldehyde in cacodylate buffer for $2 \mathrm{~h}$. The specimens were dehydrated and embedded in TAAB embedding resin (TAAB, Aldermaston). They were sectioned at $60 \mathrm{~nm}$ 
using a Leica UCT ultramicrotome (Leica Microsystems, Vienna, Austria), stained with lead citrate and uranyl acetate, and analysed with a Zeiss EM 902 transmission electron microscope (Carl Zeiss Oberkochen).

\section{RNA isolation and semi-quantitive RT-qPCR}

Total RNA was extracted using Trizol (Invitrogen, Carlsbad, CA, USA) according to the manufacturer's instructions. cDNA was synthesized using the RevertAid ${ }^{\mathrm{TM}} \mathrm{H}$ Minus first strand cDNA synthesis kit (Fermentas, St. Leon-Rot, Germany). To investigate the mRNA synthesis of collagen type I, two different primer pairs were used (col 1-1 fw: 5'-AAA CAA TGG TGC TCA GGG AC-3' and col 1-1 rev: 5'-AGG ACC AGG GAG ACC AAA CT-3'; Col 1-2 fw: 5'-CAG CAC CTT CTC TCA GAC CC-3' and Col 1-2 rev: 5'-GCA TCC TTG GTT AGG GTC AA- $3^{\prime}$ ). The mRNA of FGF1, PDGF- $\alpha$ and TGF- $\beta$, CALR, and DCN were measured with a commercially available primer assay (Qiagen, Hilden, Germany).

Semi-quantitative real-time PCR (RT-qPCR) was performed in triplicate using an ABI Prism 7000 Detection System (Applied Biosystems, Carlsbad, CA, USA). Reaction mix $(25 \mu \mathrm{l}): 1 \times \mathrm{SYBR}^{\circledR}$ Green PCR Master Mix (Invitrogen, Carlsbad, CA, USA), forward and reverse primer (1 mM each), $3 \mu 1 \mathrm{cDNA}$. Glyceraldehyde-3-phosphate dehydrogenase (GAPDH), and hypoxanthine-guanine phosphoribosyltransferase (HPRT1) served as housekeeping genes. The results are expressed as relative units based on calculation $2^{-\Delta \Delta C T}$, which yields the relative amount of target gene normalised to the endogenous control (mean of two housekeeping genes) and relative to peripheral blood mononuclear cells. The cycling protocol was as follows: one cycle of $50{ }^{\circ} \mathrm{C}$ for $2 \mathrm{~min}$ and $95{ }^{\circ} \mathrm{C}$ for $10 \mathrm{~min}$, followed by 50 cycles consisting of denaturation for $15 \mathrm{~s}$ at $95{ }^{\circ} \mathrm{C}$, annealing of primers and elongation for $1 \mathrm{~min}$ at $60{ }^{\circ} \mathrm{C}$.

\section{Statistical analysis}

All statistical analyses were performed using the Statistical Package for Social Sciences, version 17.0 (IBM, NY, USA). Differences in expression levels were analysed using the Mann-Whitney $U$ test. Spearman's correlation test was performed to examine any correlation of collagen type I expression to FGF-1, PDGF- $\alpha$, TGF- $\beta$, CALR, and DCN. A $p$ value lower than 0.05 was considered to indicate statistical significance. Expression levels are presented as means \pm standard deviation. All statistical tests were two-sided.

\section{Results}

\section{Borrelia bind to fibroblasts, invade them, and undergo extracellular cystic transformation}

Morphologic changes were identical when using BB B31 and $\mathrm{BA}$ in co-culture with fibroblasts in RPMI. Owing to better tissue preservation, the presented images are shown for the BA/ fibroblast co-culture. The first changes were noted after $24 \mathrm{~h}$ of co-culture. BA bind to fibroblast membranes, forming tethers or being surrounded by a cytoplasmic extension (Fig. 1a). Even at this stage, some Borrelia seem to be enclosed in fibroblasts, thus preserving the membranes of both Borrelia and fibroblasts. Invagination and the passage of Borrelia through the fibroblast become evident after $48 \mathrm{~h}$ (Fig. 1b). Borrelia are surrounded by a dense microfilament network (diameter $\sim 6 \mathrm{~nm}$ ) showing condensations at the fibroblast`s plasma membrane (Fig. 1b).

At all timepoints and also after $72 \mathrm{~h}$, the fibroblasts are intact, showing a prominent granulated endoplasmic reticulum (Figs. 1b, 2a, b, 4a, b). In contrast, Borrelia show enlargement of the outer membrane and some outer membrane blebs after $24 \mathrm{~h}$ (Fig. 3a), after $48 \mathrm{~h}$ abundant shedding of the outer membrane with multiple blebs and tubules in extracellular location (Fig. 3b). After 96 h, cyst and gemma formation of Borrelia is predominant (Fig. 3c, d). Degenerative changes were seen within the fibroblasts after 1 week, which hindered their assessment by electron microscopy. Fibroblasts of co-culture and control fibroblasts showed myelin bodies, lipid droplets, and autophagolysosomes, indicating that autophagic processes were taking place in the fibroblasts, both in co-cultures and in control cultures (Fig. 4a, b).

\section{Type I collagen mRNA expression is significantly increased in fibroblast/B. burgdorferi co-cultures compared to fibroblasts only}

Using two different semi-quantitative real-time PCR assays, BA and BB co-cultures were associated with a significant mRNA induction of type I collagen after 2 days compared to cultured fibroblasts (around fourfold for BA and 1.8-fold for BB for both assays, $p<0.02$ ), (Fig. 5a, b). No significant difference in type I collagen mRNA expression was detected after 7 days when comparing co-cultures with cultured fibroblasts.

\section{Growth factors that regulate collagen metabolism are significantly upregulated in fibroblast/ $B$. burgdorferi co-cultures}

mRNA expression analyses of growth factors demonstrated a significant upregulation of TGF- $\beta$ (sixfold for BA and 2.5fold for BB), CALR (ninefold for BA and 4.2-fold for BB), 


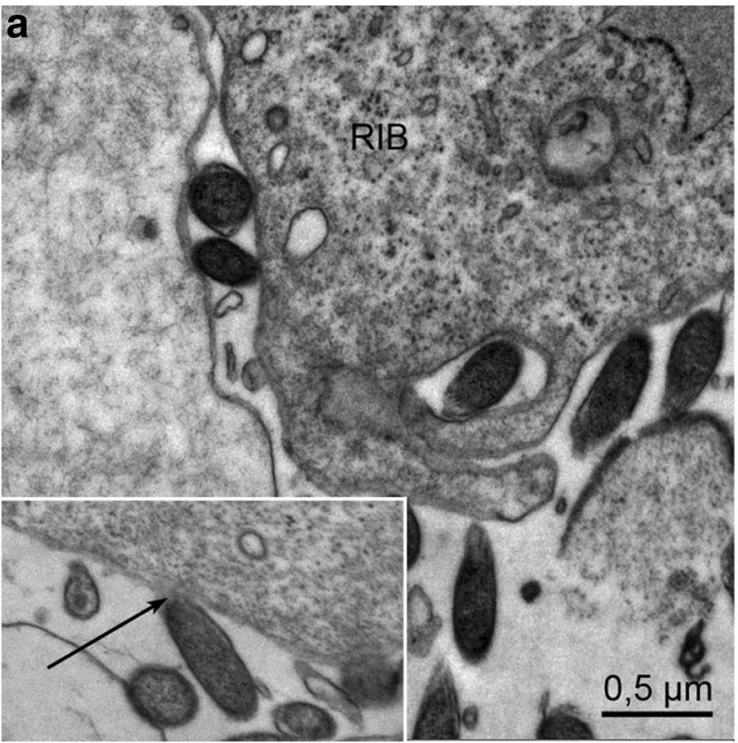

Fig. 1 a Interaction of $B$. afzelii and fibroblasts after co-culture for $24 \mathrm{~h}$ : B. afzelii bind to fibroblast membranes by tethers shown by an arrow. Borrelia seem to be invaginated in fibroblasts. Both the membranes of Borrelia and fibroblasts are intact. Ribosomes (RIB). b After $48 \mathrm{~h}$ intact, Borrelia is enclosed in a vesicle in the fibroblast's cytoplasm, thus indicating that Borrelia passes through the cyto-

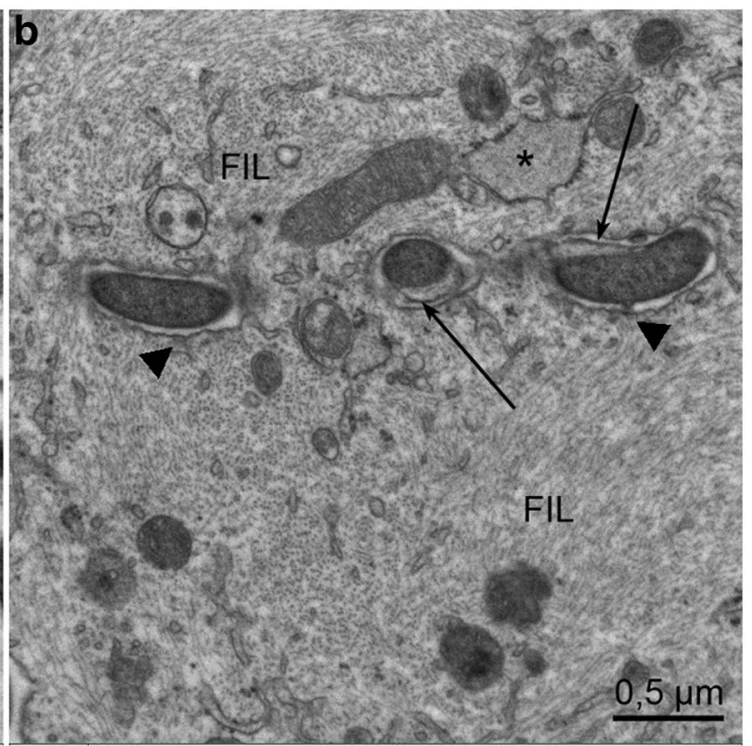

plasm. Multiple parallel and cross-sectioned filaments labelled as FIL_ probably actin microfilaments-are seen in the vicinity with condensations at the fibroblast membrane surrounding the Borrelia forming attachment plaques for the filaments marked by triangles. Outer envelope of B. afzelii is marked by arrows. Endoplasmic reticulum is labelled by an asterisk

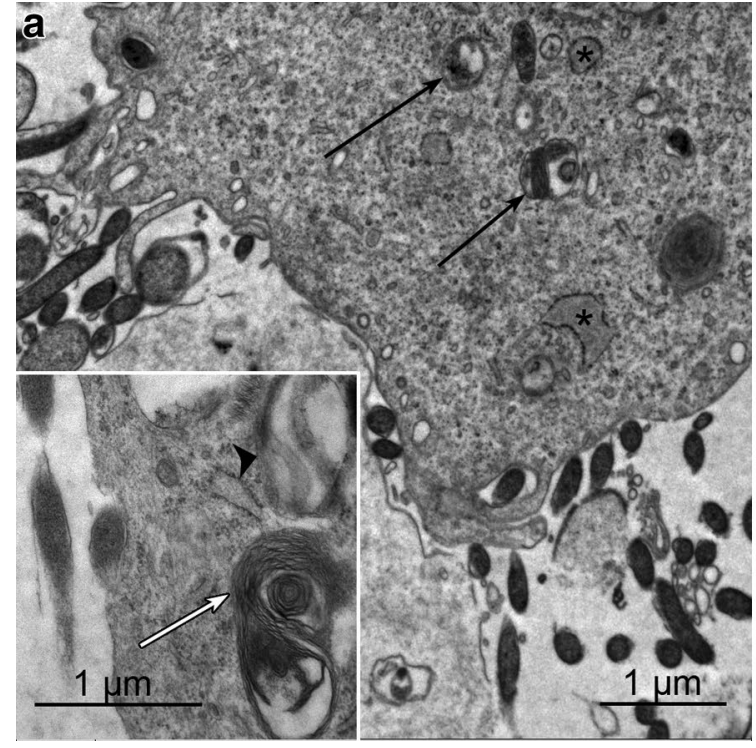

Fig. 2 After $24 \mathrm{~h}$ co-culture with B. afzelii (a) and control fibroblasts (b) exhibit autophagolysosomes shown by arrows. Inset in a: putative degenerative form of $B$. afzelii enclosed in a vacuole marked by

PDGF- $\alpha$ (sevenfold), and DCN (eightfold) in BA and BB/ fibroblast co-cultures compared to control fibroblasts, after 2 days $(p<0.01)$ (Fig. $5 \mathrm{~d})$. In contrast, a significant downregulation of FGF-1 mRNA expression (20-fold for BA and 4.5-fold for BB B31) was detected in BA and BB co-cultures

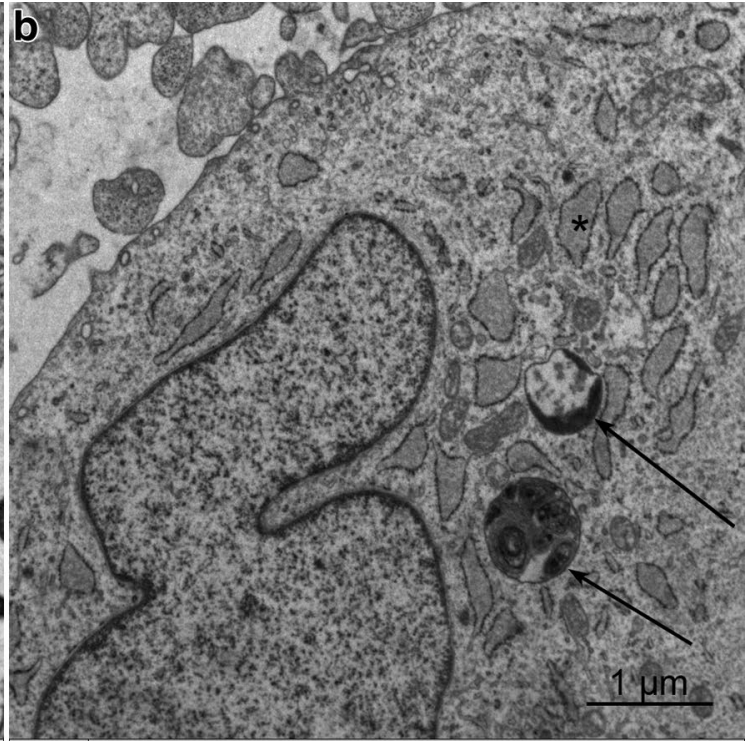

arrowhead and a myelin body labelled by an open arrow. Endoplasmic reticulum is labelled by an asterisk

compared to controls $(p<0.01)$ after 2 days (Fig. 5c). However, none of these growth factors revealed any significant difference between BA and BB co-cultures. Correlating type I collagen expression to mRNA levels of their regulating growth factors and proteins, a significant positive correlation 

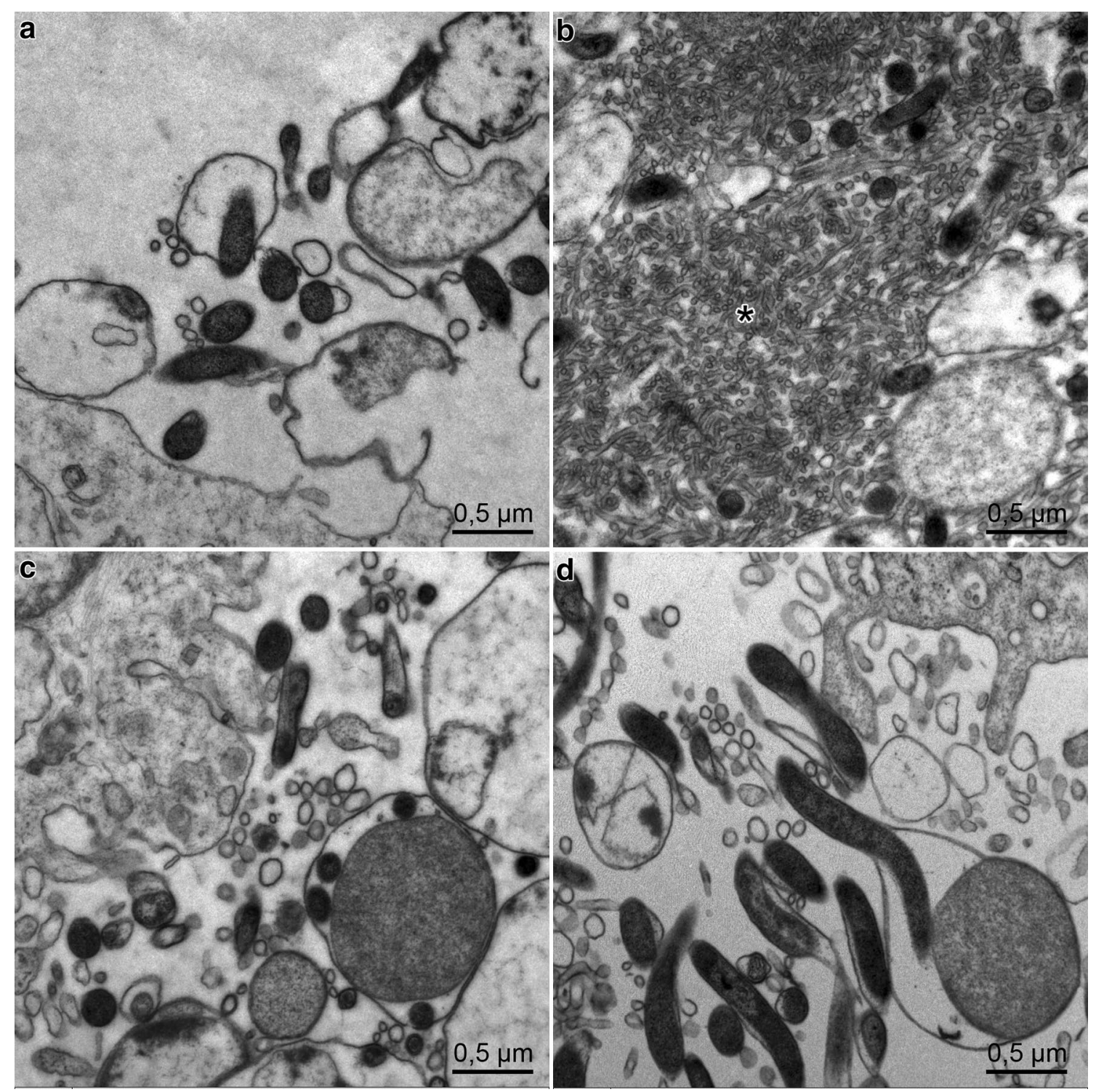

Fig. 3 Extracellular changes of B. afzelii: cyst formation, shedding of the outer membrane with numerous outer membrane blebs and tubular structures marked by an asterisk a after $24 \mathrm{~h} ; \mathbf{b}$ after $48 \mathrm{~h} ; \mathbf{c}, \mathbf{d}$ after $96 \mathrm{~h}$

of TGF- $\beta$ (Spearman's rho $0.728 p=0.026$ ), PDGF- $\alpha$ (Spearman's rho $0.703 p=0.035$ ), CALR (Spearman's rho $0.829 p=0.006$ ), and DCN (Spearman's rho $0.795 p=0.041$ ) was observed. In addition, expression levels of FGF-1 were negatively correlated with type I collagen expression (Spearman's rho $-0.705 p=0.035$ ).

\section{Discussion}

In the present study, we registered, for the first time, the excessive stimulation of collagen mRNA synthesis in fibroblast cultures when co-cultured with Borrelia. Furthermore, mRNA expression of the growth factors TGF- $\beta$, PDGF- $\alpha$,
CALR, and DCN was significantly correlated with collagen mRNA synthesis.

In agreement with the previous findings, we noted that Borrelia enter fibroblasts by forming tethers between the cell wall of the Borrelia and the cell membrane of the fibroblast, then transmigrate through these cells, and are continuously surrounded by the fibroblast's cell membrane. Klempner also studied co-cultures by electron microscopy and observed that Borrelia were internalized in a host vesicle [23]. Wu and co-workers reported that $B$. burgdorferi is able to internalise into different eukaryotic cells including endothelial cells, fibroblasts, neuronal, and neuroglial cells [51]. The entry of Borrelia was also studied in Vero cells [16]. At 24-48 h, most Vero cells had spirochetes either attached to 


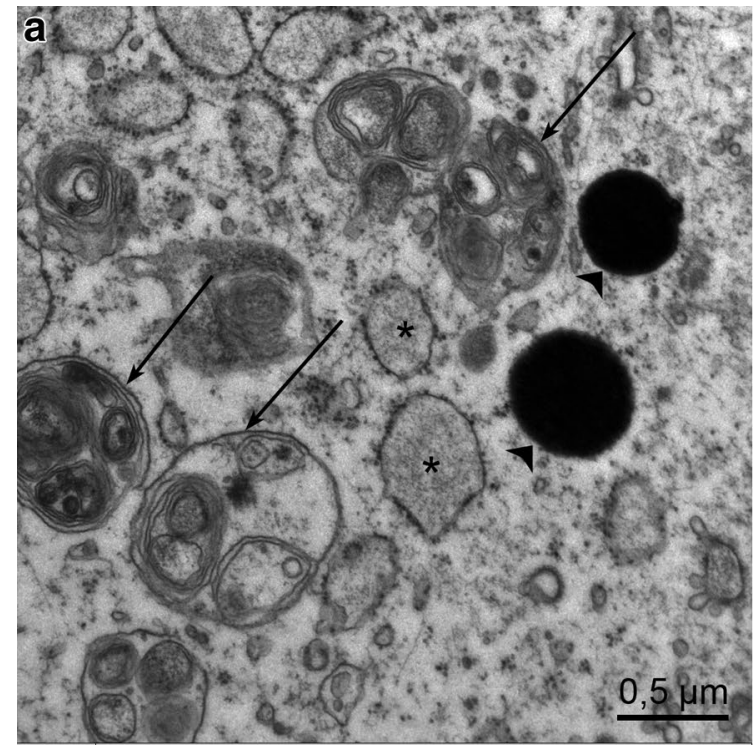

Fig. 4 a Fibroblast co-culture with $B$. afzelii after 72 h. Numerous autophagolysosomes shown by arrows and lipid droplets shown by arrowheads. b Control fibroblast after $24 \mathrm{~h}$ of co-culture with

their surface or internalized. Numerous coated pits and vesicles (120-150 $\mathrm{nm}$ diameter) were attached to the penetrating spirochetes. The uptake of Borrelia by fibroblasts differed in our study. Borrelia were attached to the cell surface or folded into the cell membrane of fibroblasts, as noted in synovial cells [12].

Fibronectin has been shown to mediate Borrelia attachment $[9,24]$. However, the invasion of Borrelia requires $\beta-1$ integrins [51]. Fibrinogen binding by Borrelia may create a bridge for binding integrins [18]. The decorin-binding protein A is important for tethering, as observed on endothelial cells. Furthermore, P66, a porin with adhesive capability, may support the transmigration of Borrelia and intracellular invasion [18]. The cytoskeleton is responsible for internalisation of bacteria, as observed in a Bacillus anthracis/fibroblasts cell line [39]. Wu et al. suggested that the reorganisation of actin filaments and Src family kinases is necessary for internalisation [51]. We found densely packed filaments in close apposition to Borrelia within fibroblasts, probably representing actin filaments.

It has been reported that the internalisation of B. burgdorferi does not alter the viability of mammalian cells [51]. The viability of fibroblasts was similar in both, co-cultures and control cultures of fibroblasts alone; after 1 week autophagolysosomes were found in both cultures.

Borrelia are known to undergo pathologic changes such as shedding of the outer envelope and cystic malformation under adverse conditions like exposure to penicillin or deprivation of nutrients in the culture fluid [22, 51]. In our study as well, Borrelia showed typical bleb and gemma

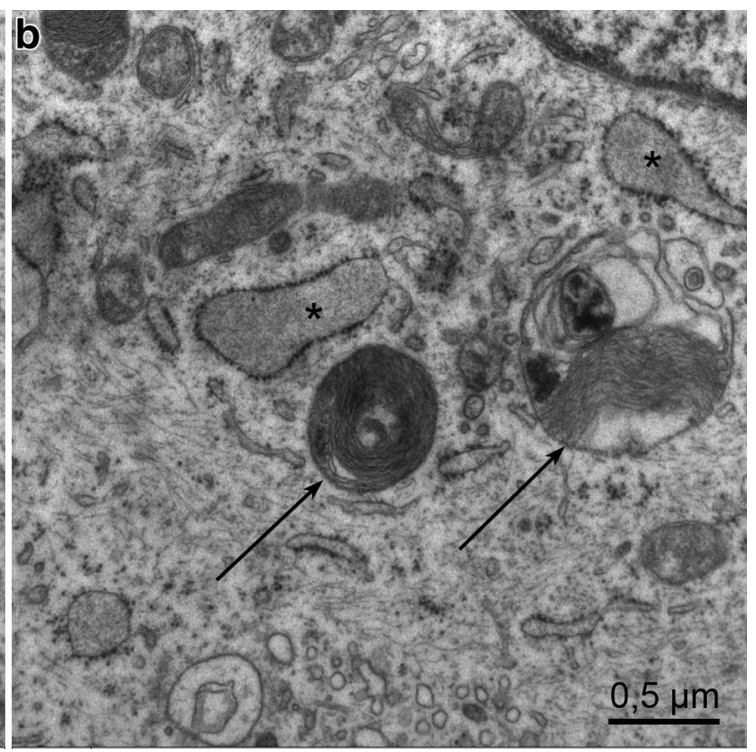

autophagolysosomes of different shapes marked by arrows. Endoplasmic reticulum is labelled by an asterisk

formation, and cystic expansion as a sign of malnutrition. The latter might have been induced by co-culture in RPMI and deprivation of important nutrients for the growth of Borrelia, as is true of the BSK-H medium. We co-cultured fibroblasts with Borrelia according to a previous protocol by Klempner et al., which suggests RPMI as culture medium [23].

Both Borrelia species BB and BA are associated with similar morphologic changes. However, the role of the genospecies-specific immune response has not been studied in detail. In the recent past, human dermal fibroblasts co-cultured with the three main B. burgdorferi sensu lato species showed a homogenous inflammatory gene profile with similar transcriptional profiles and no species-specific fingerprint of transcriptional changes in fibroblasts, including a common core of chemokines/cytokines and interferonrelated genes [30].

We found a considerable upregulation of TGF- $\beta$ mRNA synthesis in fibroblasts co-cultured with BA, resulting in a fourfold increase of collagen type I mRNA and a 1.8fold increase after exposure to BB compared to control fibroblasts.

The distribution of collagens I and III in the skin varies in the different skin layers with substantial differences in expression of mRNA for type I and III procollagen [4]. In morphea, fibroblast cell lines produced increased amounts of type I and type III collagens, but the ratios of type I and type III collagens remained relatively unchanged in all the cultures, suggesting that they have undergone a coordinated activation of collagen synthesis at transcriptional level [47]. 
a

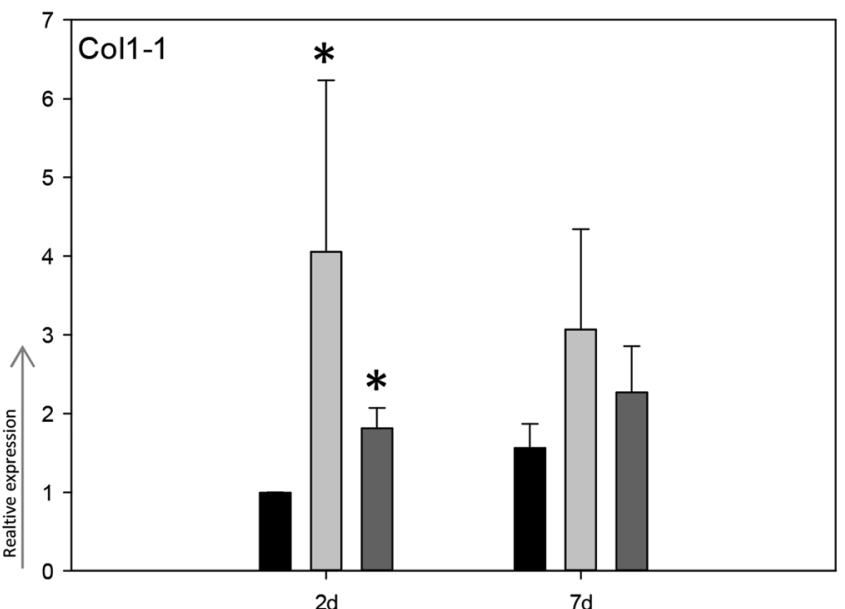

$2 d$ b

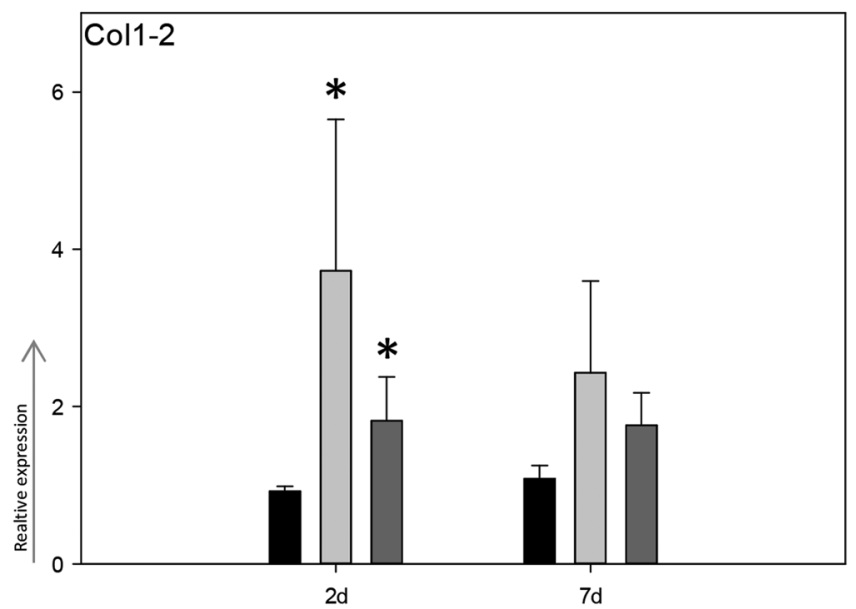

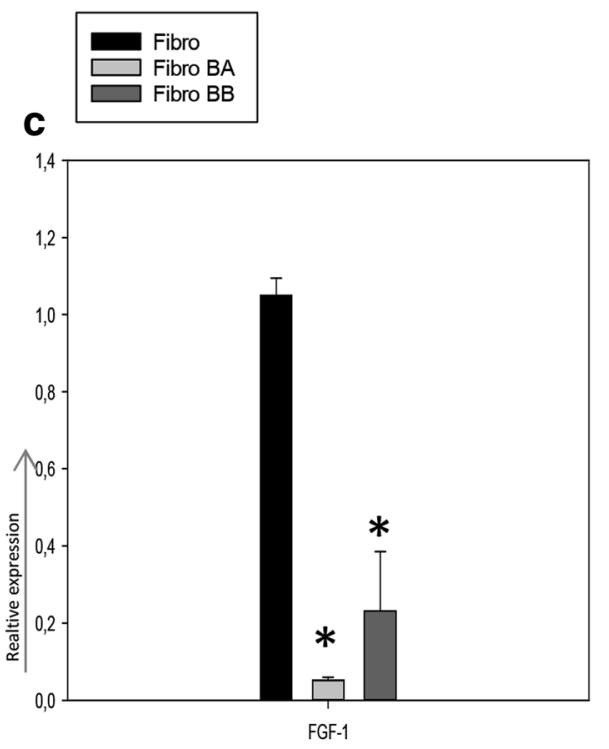

d

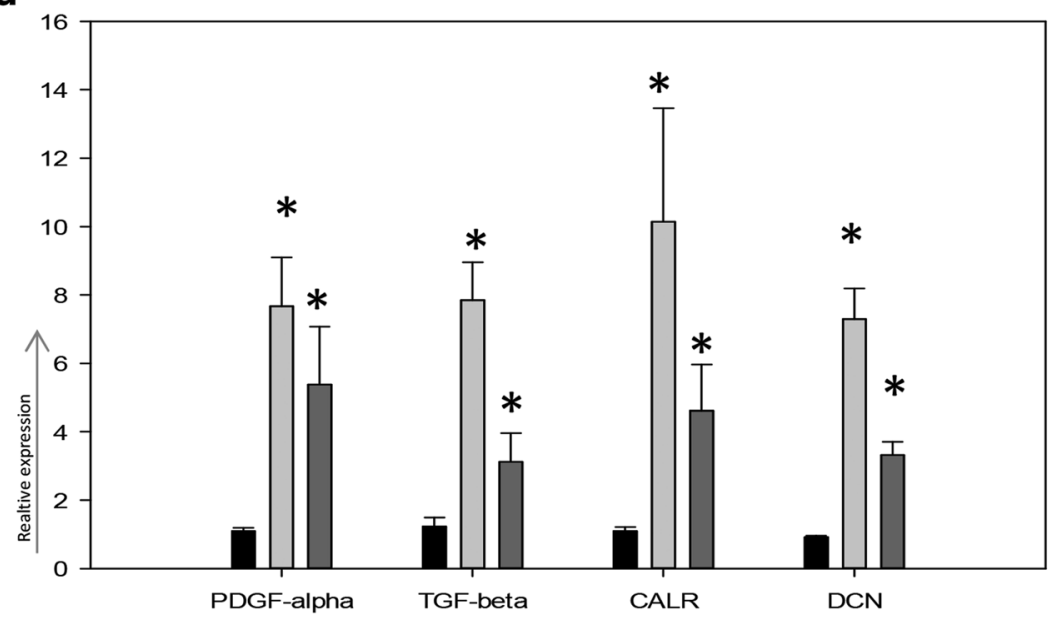

Fig. 5 Type 1 collagen, FGF-1, PDGF-alpha, TGF beta, CALR and DCN expression in fibroblasts (Fibro), fibroblasts co-cultured with B. afzelii (Fibro BA) and fibroblasts co-cultured with B. burgdorferi sensu stricto B31 (Fibro BB). a, b Type 1 collagen expression of four technical replicates of the two PCR assays (Col1-1 and Col1-2). c

We only investigated type I collagen mRNA synthesis in our co-culture experiments, because type I collagen is the predominant type in normal and sclerotic skin [2]. Human fibroblasts in culture synthesize both type I and type III collagen, with type I accounting for 70-90\% of the total. The proportion of type III collagen is dependent on culture conditions and differs in patients with certain connective tissue diseases. Whereas the proportion of type III collagen in normal fibroblast cell lines is increased at high cell density, cells from patients with systemic sclerosis grow at a lower density than control cells and have a reduced type III/I ratio. It could well be that fibroblasts
FGF-1 expression of three biological replicates. d PDGF-alpha, TGF beta, CALR and DCN expression of three biological replicates. Each bar represents the mean expression of error bars indicate standard error of the mean. Stars indicate significant deregulation compared to controls

exposed to $B$. burgdorferi produce type III collagen in a different way we did not look for.

TGF- $\beta$ is known to markedly enhance the production of collagen types I and III. In human dermal fibroblasts exposed to TGF- $\beta$, Varga et al. observed a two to threefold increase in the quantities of collagen measured by hydroxy $\left[{ }^{14} \mathrm{C}\right]$ proline synthesis [43]. Total RNA extracted from skin biopsies of systemic sclerosis showed a greater than threefold elevation of collagen I mRNA levels compared to controls [17]. TGF- $\beta$ is a key regulator of extracellular matrix assembly and remodelling. Elevated TGF- $\beta$ expression in the affected organs correlates with connective tissue deposits in 
the process of fibrosis [45]. TGF- $\beta 2$ mRNA was found to be co-localised with collagen I expression in patients with the inflammatory stage of systemic sclerosis [26]. Similarly, decorin works together with TGF- $\beta$ in controlling fibrosis [14].

Collagen turnover is regulated by local growth factors that stimulate fibroblasts to produce extracellular matrix proteins. Chaperones play an important role in maintaining the quality of protein processing in the endoplasmic reticulum (ER) [13]. In our study, co-cultured and control fibroblasts were rich in granular ER, indicating active RNA synthesis [31]. Calreticulin is an ER chaperone upregulated by ER stress in fibrotic tissue [54]. CALR regulates type I collagen transcription and processing into the extracellular matrix (ECM). Fibroblasts with increased CALR expression enhance the response to TGF- $B$. A correlation has been reported between CALR upregulation and the progression of fibrosis. CALR was also upregulated in a bleomycin-induced animal model of lung fibrosis [27]. In our Borrelia/fibroblast co-culture, the production of CALR was significantly elevated at the mRNA level, thus enhancing collagen synthesis.

DCN is a key regulatory molecule in collagen fibril assembly [53]. The role of DCN in scleroderma was studied by Vuorio et al. Fibroblasts were cultured from affected skin areas of patients with systemic and localised scleroderma, and investigated in regard of mRNA levels of TGF- $\beta$ and DCN. Whereas TGF- $B$ mRNA was found to be slightly elevated in fibroblasts, DCN mRNA showed marked variations in cell lines and was not correlated with collagen mRNA [46]. In our study, DCN mRNA was significantly overexpressed in Borrelia/fibroblast co-cultures compared to fibroblast cultures. BB expresses DNC binding proteins at the outer surface; these are lipoproteins which bind decorin and glycosaminoglycans [41]. In laboratory mice with chronic infection, Borrelia were found to absolutely co-localise with decorin but not with collagen I [20]. In an autopsy study, B. burgdorferi was found in the cardiac interstitium of five patients who experienced sudden cardiac death. B. burgdorferi was associated with collagen fibres and co-localised with DCN as well, as shown by immunohistochemistry [32].

PDGF is involved in fibrosis associated with systemic sclerosis. PDGF receptors are expressed on collagensecreting fibroblasts [21]. Resident dermal fibroblasts and/ or myofibroblasts promote skin fibrosis by overproducing the collagen-rich extracellular matrix. PDGF- $\alpha$ mRNA was significantly higher in Borrelia/fibroblast co-cultures than in control fibroblasts.

Compared to the action of different growth factors inducing increased collagen RNA synthesis, FGF-1 had the opposite effect. A significant down-regulation of FGF-1 mRNA expression in co-cultures was found to be negatively correlated with collagen I expression. This confirmed previous data reported by Ichiki et al., who showed that FGF is a potent inhibitor of basal and TGF$\beta$-stimulated collagen expression in human skin fibroblasts [19].

To our knowledge, the role of different growth factors in collagen synthesis has not yet been investigated in LB. One of the major limitations of the present study was that no data were available for secreted growth factors at the protein level. The results reported here must be validated in further experiments. We also do not know whether autocrine secretion of growth factors in fibroblasts can influence fibroblast activity and increased collagen gene expression, such as with bFGF, PDGF, or ascorbic acid which is a constituent of the culture medium $[1,34$, 50]. Because these factors are part of the media, we suppose that they are working together with paracrine and autocrine stimulation of fibroblasts. Besides, cytokines or growth factors produced by the interaction of Borrelia with the immune system may further influence collagen gene expression which, to our knowledge, has not yet been studied in LB.

Our findings support the hypothesis that skin fibrosis can be triggered by Borrelia, presumably BA and to a lesser extent also BB B31, by stimulating several profibrotic molecules such as TGF- $\beta$, CALR, DCN, PDGF- $\alpha$. All of these, with the exception of FGF, are positively correlated with mRNA collagen expression. The difference in the levels of collagen mRNA which were considerably but not significantly higher in BA co-culture compared to BB may suggest a higher risk for fibrosis in countries with predominance of $B$. afzelii. Further strains of $B$. burgdorferi sensu lato should be co-cultured with fibroblasts, also of other tissues like tendon, and fibroblast cultures from sclerotic lesions of circumscribed scleroderma. Whether these findings may have implications for clinical entities such as circumscribed scleroderma, lichen sclerosus et atrophicus, carpal tunnel syndrome, or the trigger finger, remains to be seen. The exact mechanism of action in vivo is unknown and will have to be studied in further in vitro studies and animal models.

Acknowledgements We greatly acknowledge the support of Ilse Wendelin and Doris Stünzner, Institute of Hygiene and Microbiology in culturing borrelia; Doris Stünzner for isolating B. burgdorferi from skin biopsies and genospecies typing; Elisabeth Bock and Maria Anna Pabst, Institute of Cell Biology, for EM specimen preparation and analysis; and the staff at the Medical University of Graz. We are indebted to Beate Eckes for technical instructions on collagen PCR, and Thomas Krieg, both at the Department of Dermatology, University of Cologne, Germany, for their valuable discussions and comments.

\section{Compliance with ethical standards}

Funding Open access funding provided by Medical University of Graz.

Conflict of interest The authors declare that they have no conflict of interest. 
Ethical approval This article does not contain any studies with human participants or animals performed by any of the authors.

Open Access This article is distributed under the terms of the Creative Commons Attribution 4.0 International License (http://creativecommons.org/licenses/by/4.0/), which permits unrestricted use, distribution, and reproduction in any medium, provided you give appropriate credit to the original author(s) and the source, provide a link to the Creative Commons license, and indicate if changes were made.

\section{References}

1. Abdian N, Ghasemi-Dehkordi P, Hashemzadeh-Chaleshtori M, Ganji-Arjenaki M, Doosti A, Amiri B (2015) Comparison of human dermal fibroblasts (HDFs) growth rate in culture media supplemented with or without basic fibroblast growth factor (bFGF). Cell Tissue Bank 16:487-495

2. Abe S, Steinmann BU, Wahl LM, Martin GR (1979) High cell density alters the ratio of type III to I collagen synthesis by fibroblasts. Nature 279:442-444

3. Aberer E, Klade H, Stanek G, Gebhart W (1991) Borrelia burgdorferi and different types of morphea. Dermatologica 182:145-154

4. Ali-Bahar M, Bauer B, Tredget EE, Ghahary A (2004) Dermal fibroblasts from different layers of human skin are heterogeneous in expression of collagenase and types I and III procollagen mRNA. Wound Repair Regen 12:175-182

5. Breier F, Khanakah G, Stanek G, Kunz G, Aberer E, Schmidt B, Tappeiner $G$ (2001) Isolation and polymerase chain reaction typing of Borrelia afzelii from a skin lesion in a seronegative patient with generalized ulcerating bullous lichen sclerosus et atrophicus. Br J Dermatol 144:387-392

6. Breier FH, Aberer E, Stanek G, Khanakaha G, Schlick A, Tappeiner G (1999) Isolation of Borrelia afzelii from circumscribed scleroderma. Br J Dermatol 140:925-930

7. Cadavid D, Bai Y, Hodzic E, Narayan K, Barthold SW, Pachner AR (2004) Cardiac involvement in non-human primates infected with the Lyme disease spirochete Borrelia burgdorferi. Lab Invest 84:1439-1450

8. Chujo S, Shirasaki F, Kondo-Miyazaki M, Ikawa Y, Takehara K (2009) Role of connective tissue growth factor and its interaction with basic fibroblast growth factor and macrophage chemoattractant protein-1 in skin fibrosis. J Cell Physiol 220:189-195

9. Coburn J, Fischer JR, Leong JM (2005) Solving a sticky problem: new genetic approaches to host cell adhesion by the Lyme disease spirochete. Mol Microbio 57:1182-1195

10. Elias AC, Ricci MD, Rodriguez LH, Pinto SD, Giglio JS, Baracat EC (2015) The biopsychosocial spiritual model applied to the treatment of women with breast cancer, through RIME intervention (relaxation, mental images, spirituality). Complement Ther Clin Pract 21:1-6

11. Fujiwara H, Fujiwara K, Hashimoto K, Mehregan AH, Schaumburg-Lever G, Lange R, Schempp C, Gollnick H (1997) Detection of Borrelia burgdorferi DNA (B. garinii or B. afzelii) in morphea and lichen sclerosus et atrophicus tissues of German and Japanese but not of US patients. Arch Dermatol 133:41-44

12. Girschick HJ, Huppertz HI, Russmann H, Krenn V, Karch H (1996) Intracellular persistence of Borrelia burgdorferi in human synovial cells. Rheumatol Int 16:125-132

13. Gold L, Williams D, Groenendyk J, Michalak M, Eggleton P (2015) Unfolding the complexities of ER chaperones in health and disease: report on the 11th international calreticulin workshop. Cell Stress Chaperon 20:875-883
14. Harper JR, Spiro RC, Gaarde WA, Tamura RN, Pierschbacher MD, Noble NA, Stecker KK, Border WA (1994) Role of transforming growth factor beta and decorin in controlling fibrosis. Methods Enzymol 245:241-254

15. Haupl T, Hahn G, Rittig M, Krause A, Schoerner C, Schonherr U, Kalden JR, Burmester GR (1993) Persistence of Borrelia burgdorferi in ligamentous tissue from a patient with chronic Lyme borreliosis. Arthritis Rheum 36:1621-1626

16. Hechemy KE, Samsonoff WA, Harris HL, McKee M (1992) Adherence and entry of Borrelia burgdorferi in Vero cells. J Med Microbiol 36:229-238

17. Herrmann K, Heckmann M, Kulozik M, Haustein UF, Krieg T (1991) Steady-state mRNA levels of collagens I, III, fibronectin, and collagenase in skin biopsies of systemic sclerosis patients. J Invest Dermatol 97:219-222

18. Hyde JA (2017) Borrelia burgdorferi keeps moving and carries on: a review of borrelial dissemination and invasion. Front Immunol 8:114

19. Ichiki Y, Smith EA, LeRoy EC, Trojanowska M (1997) Basic fibroblast growth factor inhibits basal and transforming growth factor-beta induced collagen alpha 2(I) gene expression in scleroderma and normal fibroblasts. J Rheumatol 24:90-95

20. Imai DM, Feng S, Hodzic E, Barthold SW (2013) Dynamics of connective-tissue localization during chronic Borrelia burgdorferi infection. Lab Invest 93:900-910

21. Iwayama T, Olson LE (2013) Involvement of PDGF in fibrosis and scleroderma: recent insights from animal models and potential therapeutic opportunities. Curr Rheumatol Rep 15:304

22. Kersten A, Poitschek C, Rauch S, Aberer E (1995) Effects of penicillin, ceftriaxone, and doxycycline on morphology of Borrelia burgdorferi. Antimicrob Agents Chemother 39:1127-1133

23. Klempner MS, Noring R, Rogers RA (1993) Invasion of human skin fibroblasts by the Lyme disease spirochete, Borrelia burgdorferi. J Infect Dis 167:1074-1081

24. Kopp PA, Schmitt M, Wellensiek HJ, Blobel H (1995) Isolation and characterization of fibronectin-binding sites of Borrelia garinii N34. Infect Immun 63:3804-3808

25. Krieg T, Braun-Falco O, Perlish JS, Fleischmajer R (1983) Collagen synthesis in generalized morphea. Arch Dermatol Res 275:393-396

26. Kulozik M, Hogg A, Lankat-Buttgereit B, Krieg T (1990) Colocalization of transforming growth factor beta 2 with alpha $1(\mathrm{I})$ procollagen mRNA in tissue sections of patients with systemic sclerosis. J Clin Invest 86:917-922

27. Kypreou KP, Kavvadas P, Karamessinis P, Peroulis M, Alberti A, Sideras P, Psarras S, Capetanaki Y, Politis PK, Charonis AS (2008) Altered expression of calreticulin during the development of fibrosis. Proteomics 8:2407-2419

28. Liang FT, Brown EL, Wang T, Iozzo RV, Fikrig E (2004) Protective niche for Borrelia burgdorferi to evade humoral immunity. Am J Pathol 165:977-985

29. Lunemann JD, Krause A (2003) Heterogeneity of Borrelia burgdorferi: etiopathogenetic relevance and clinical implications. Z Rheumatol 62:148-154

30. Meddeb M, Carpentier W, Cagnard N, Nadaud S, Grillon A, Barthel C, De Martino SJ, Jaulhac B, Boulanger N, Schramm $F$ (2016) Homogeneous inflammatory gene profiles induced in human dermal fibroblasts in response to the three main species of Borrelia burgdorferi sensu lato. PLoS One 11:e0164117

31. Movat HZ, Fernando NV (1962) The fine structure of connective tissue. I. The fibroblast. Exp Mol Pathol 1:509-534

32. Muehlenbachs A, Bollweg BC, Schulz TJ, Forrester JD, DeLeon Carnes M, Molins C, Ray GS, Cummings PM, Ritter JM, Blau DM, Andrew TA, Prial M, Ng DL, Prahlow JA, Sanders JH, Shieh WJ, Paddock CD, Schriefer ME, Mead P, Zaki SR (2016) Cardiac tropism of Borrelia burgdorferi: an autopsy study of 
sudden cardiac death associated with lyme carditis. Am J Pathol 186:1195-1205

33. Parveen N, Leong JM (2000) Identification of a candidate glycosaminoglycan-binding adhesin of the Lyme disease spirochete Borrelia burgdorferi. Mol Microbiol 35:1220-1234

34. Phillips CL, Combs SB, Pinnell SR (1994) Effects of ascorbic acid on proliferation and collagen synthesis in relation to the donor age of human dermal fibroblasts. J Invest Dermatol 103:228-232

35. Picken RN, Strle F, Picken MM, Ruzic-Sabljic E, Maraspin V, Lotric-Furlan S, Cimperman J (1998) Identification of three species of Borrelia burgdorferi sensu lato (B. burgdorferi sensu stricto, B. garinii, and B. afzelii) among isolates from acrodermatitis chronica atrophicans lesions. J Invest Dermatol 110:211-214

36. Pollack RJ, Telford SR 3rd, Spielman A (1993) Standardization of medium for culturing Lyme disease spirochetes. J Clin Microbiol 31:1251-1255

37. Ramesh G, Alvarez AL, Roberts ED, Dennis VA, Lasater BL, Alvarez X, Philipp MT (2003) Pathogenesis of Lyme neuroborreliosis: Borrelia burgdorferi lipoproteins induce both proliferation and apoptosis in rhesus monkey astrocytes. Eur J Immunol 33:2539-2550

38. Roberts ED, Bohm RP Jr, Lowrie RC Jr, Habicht G, Katona L, Piesman J, Philipp MT (1998) Pathogenesis of Lyme neuroborreliosis in the rhesus monkey: the early disseminated and chronic phases of disease in the peripheral nervous system. J Infect Dis 178:722-732

39. Russell BH, Vasan R, Keene DR, Xu Y (2007) Bacillus anthracis internalization by human fibroblasts and epithelial cells. Cell Microbiol 9:1262-1274

40. Sepp N, Schmutzhard E, Fritsch P (1988) Shulman syndrome associated with Borrelia burgdorferi and complicated by carpal tunnel syndrome. J Am Acad Dermatol 18:1361-1362

41. Shi Y, Xu Q, McShan K, Liang FT (2008) Both decorin-binding proteins A and B are critical for the overall virulence of Borrelia burgdorferi. Infect Immun 76:1239-1246

42. Stünzner D, Hubalek Z, Halouzka J, Postic D, Pierer K, Marth E (1998) Prevalence of Borrelia burgdorferi s.I. in Ixodes ricinus ticks from Styria (Austria) and species identification by PCR-RFLP analysis. Zentralblatt fur Bakteriologie (IJMM) 288:471-478

43. Varga J, Rosenbloom J, Jimenez SA (1987) Transforming growth factor beta (TGF beta) causes a persistent increase in steady-state amounts of type I and type III collagen and fibronectin mRNAs in normal human dermal fibroblasts. Biochem J 247:597-604

44. Verrecchia F, Laboureau J, Verola O, Roos N, Porcher R, Bruneval P, Ertault M, Tiev K, Michel L, Mauviel A, Farge D (2007) Skin involvement in scleroderma-where histological and clinical scores meet. Rheumatology 46:833-841

45. Verrecchia F, Mauviel A (2004) TGF-beta and TNF-alpha: antagonistic cytokines controlling type I collagen gene expression. Cell Signal 16:873-880

46. Vuorio T, Kahari VM, Black C, Vuorio E (1991) Expression of osteonectin, decorin, and transforming growth factor-beta 1 genes in fibroblasts cultured from patients with systemic sclerosis and morphea. J Rheumatol 18:247-251

47. Vuorio T, Makela JK, Kahari VM, Vuorio E (1987) Coordinated regulation of type I and type III collagen production and mRNA levels of pro alpha $1(\mathrm{I})$ and pro alpha 2(I) collagen in cultured morphea fibroblasts. Arch Dermatol Res 279:154-160

48. Wackernagel A, Bergmann AR, Aberer E (2005) Acute exacerbation of systemic scleroderma in Borrelia burgdorferi infection. $\mathrm{J}$ Eur Acad Dermatol Venereol 19:93-96

49. Wels C, Joshi S, Koefinger P, Bergler H, Schaider H (2011) Transcriptional activation of ZEB1 by Slug leads to cooperative regulation of the epithelial-mesenchymal transition-like phenotype in melanoma. J Invest Dermatol 131:1877-1885

50. Wong T, McGrath JA, Navsaria H (2007) The role of fibroblasts in tissue engineering and regeneration. Br J Dermatol 156:1149-1155

51. Wu J, Weening EH, Faske JB, Hook M, Skare JT (2011) Invasion of eukaryotic cells by Borrelia burgdorferi requires beta(1) integrins and Src kinase activity. Infect Immun 79:1338-1348

52. Zambrano MC, Beklemisheva AA, Bryksin AV, Newman SA, Cabello FC (2004) Borrelia burgdorferi binds to, invades, and colonizes native type I collagen lattices. Infect Immun 72:3138-3146

53. Zhang G, Ezura Y, Chervoneva I, Robinson PS, Beason DP, Carine ET, Soslowsky LJ, Iozzo RV, Birk DE (2006) Decorin regulates assembly of collagen fibrils and acquisition of biomechanical properties during tendon development. J Cell Biochem 98:1436-1449

54. Zimmerman KA, Graham LV, Pallero MA, Murphy-Ullrich JE (2013) Calreticulin regulates transforming growth factor-betastimulated extracellular matrix production. J Biol Chem 288:14584-14598 